\title{
TIME-TO-ARRIVAL AND USEFUL FIELD OF VIEW: ASSOCIATIONS WITH REPORTED DRIVING DIFFICULTIES AMONG OLDER ADULTS
}

\author{
José F. Marmeleira, ${ }^{1}$ Inês S. Ferreira, ${ }^{2}$ \\ Mário B. Godinho, ${ }^{3}$ Orlando M. Fernandes ${ }^{1}$ \\ ${ }^{1}$ Departamento de Desporto e Saúde \\ University of Évora, Portugal \\ E-mail: jmarmel@uevora.pt \\ ${ }^{2}$ Faculdade de Psicologia e de Ciências da Educação \\ University of Coimbra, Portugal \\ ${ }^{3}$ Faculdade de Motricidade Humana, \\ Technical University of Lisbon, Portugal
}

\begin{abstract}
Summary: The purpose of this study was to determine self-reported driving difficulties related to time-to-arrival (TTA) and/or useful field of view (UFOV), to identify associations between TTA and UFOV, and to compare the TTA estimated accuracy between different vehicle speed approaches. Thirty-eight male and female active drivers ages 61 to 81 years $(70,2 \pm 5,0$ years) participated in this study. The $\mathrm{UFOV}^{\circledR}$ test was applied. TTA was studied with a removal paradigm, using two vehicle speeds $(50$ and $70 \mathrm{~km} / \mathrm{h}$ ) for data collection. A driving habits questionnaire was administered, including items concerning driving difficulties. Results showed that lower performances in divided attention (subtest 2 of $\mathrm{UFOV}^{\circledR}$ ) were significantly associated with reported difficulties in hightraffic intersections, trouble seeing the signs in time to respond to them, and problems judging the speed or distance of an approaching vehicle. Concerning TTA, greater response bias and/or greater response inconsistency were significantly associated with difficulties in overtaking other cars, difficulties seeing the signs in time to react to them, difficulties in the speed or distance perception of an oncoming vehicle. Low associations were found between UFOV and TTA measures. TTA estimated accuracy increased when the vehicle traveled at higher speeds. We concluded that both TTA and UFOV seem to be important measures to identify driving difficulties in older adults. The TTA could be relevant in measuring specific aspects of visual information processing in addition to other instruments like $\mathrm{UFOV}^{\circledR}$.
\end{abstract}

\section{INTRODUCTION}

Considering the data on road accidents and the demographic evolution, namely the increase of drivers 65 years old or more, researchers and diverse public and private entities are showing an increasing interest in issues associated with fitness to drive in elderly drivers. The number of accidents, when we consider distances driven, is bigger for aged drivers (65 years old or more) than for all other drivers (Guerrier et al., 1999; Preusser et al., 1998). Simultaneously, the elderly also have a bigger risk of injury or death in case of a traffic accident (McGwin et al., 2000).

Diverse perceptive, cognitive and motor factors have been associated with driving difficulties and accident incidence in older adults. Concerning visual perception, visual function tests alone 
have demonstrated little sensitivity to identify risky drivers (Anstey et al., 2005; Owsley et al., 1998). The $\mathrm{UFOV}^{\circledR}$ test (Ball \& Owsley, 1993), which combines the evaluation of visual processing speed, selective and divided visual attention, was identified as a valid and reliable index of driving performance and safety in older adults (Anstey et al., 2005; Clay et al., 2005; Sims et al., 2000). Evidence showed that $\mathrm{UFOV}^{\circledR}$ test performance relies on higher-order cognitive abilities, as well as on visual sensory function (Owsley et al., 1995).

Speed perception and motion perception have also been pointed to as important abilities for safe driving (Hesketh \& Godley, 2002; Manser \& Hancock, 1996; Raghuram \& Lakshminarayanan, 2006; Staplin \& Lyles, 1992). Correctly perceiving the speed, and hence the distance and "time away" of an approaching vehicle, is a very important skill in manoeuvres where one has to turn across oncoming traffic (Hesketh \& Godley, 2002; McGwin \& Brown, 1999; Skaar et al., 2003). It's relevant that a high proportion of older drivers' accidents occur in intersections when entering traffic or crossing a main road (Mayhew et al., 2006). A deficit in accurate speed perception of oncoming vehicles judged by TTA could hinder the adequate decision in those manoeuvres.

To our knowledge, few studies have examined simultaneously the associations of UFOV and TTA with driving. A recent work by Raghuram and Lakshminarayanan (2006) concluded that the association between UFOV and calculated motion scores was not significant, indicating that probably different aspects of visual information processing crucial to driving behavior are being tapped by these two paradigms. In this context, it's important to explore the possibility of how TTA or other motion perception tasks could contribute to identifying potential subjects who are at risk while driving.

\section{METHODS}

\section{Participants}

Thirty-eight active drivers (16 females and 22 males) with ages between 61 and $81(70,2 \pm 5,0$ years) participated in this study. The participants were voluntarily recruited in the region of Lisbon, Portugal, from two Senior Universities and a University Health Program for older adults. Participants were contacted by phone or personally; $26 \%$ completed elementary school, $32 \%$ completed middle and high school, and $42 \%$ were university graduates. All participants currently drive ( $\geq 2$ days a week) and had a driver's license for over 10 years

\section{Procedures}

This study was conducted simultaneously with another investigation concerning cognitive factors related to self-reported difficulties in older drivers. The evaluations were realized individually at the Universities where the participants were recruited. The assessment of each person took approximately three hours.

The three subtests of $\mathrm{UFOV}^{\circledR}$ (PC version) were administered to measure speed of visual processing (subtest 1), divided attention (subtest 2) and selective attention (subtest 3). The first subtest requires the identification of a target (silhouette of a car or truck) presented in a central 
fixation box. The second subtest measures divided attention and involves identification of the central target along with localization of a simultaneous peripheral target (silhouette of a car or truck). The third subtest consists of these same two tasks, but also includes visual distracters (triangles of the same size and luminance as the targets) that fill the rest of the visual display.

TTA was studied using a removal paradigm: drivers saw a video projection (without sound) of an oncoming vehicle from the right way and had to press a response button (PC) when they judged the vehicle reached a previously established point on the road. After 3 practice scenes, participants judged 15 approaches: 10 were used for data collection and 5 were used as distracters. For data collection the car traveled either at 50 or $70 \mathrm{~km} / \mathrm{h}$, appeared during $7 \mathrm{sec}$, and was removed from the scenario $3 \mathrm{sec}$ before reaching the arrival point. The distracter scene consisted of an approaching car at $60 \mathrm{~km} / \mathrm{h}$ with a display time of $6 \mathrm{sec}$ and a removal time of 1.5 sec. In real conditions, the speed of each car approach was checked using a GPS Tomtom Go 700. Accuracy in performance (absolute error, AE), response bias (constant error, $\mathrm{CE}$ ) and response consistency (variable error, VE) were registered.

Information on demographic characteristics, medical conditions, physical functions and driving difficulties and habits were collected by separate questionnaires. Visual Acuity was measured with a Snellen Chart. A Driving Habits Questionnaire was adapted from Owsley et al. (1999), including more items concerning difficulties driving in specific situations (e.g., at busy intersections). Difficulties were self-reported in a dichotomic way (yes or no).

The Logistic Regression method was used to assess the relationships of self-reported driving difficulties (dependent variables) with UFOV and TTA (independent variables). For this purpose, each subtest of UFOV ${ }^{\circledR}$ and each measure of TTA was tested separately as an independent variable for every difficulty studied. To control for effects of gender, age and visual acuity, they were also included as covariates in each logistic regression performed. Backward Stepwise was the selected method. Spearman's rank correlation coefficient was calculated to study the associations between TTA and UFOV measures. The Wilcoxon signed-rank test was used to compare the results of TTA measures from two different vehicle speed approaches. For all statistical analysis significance level was set at $\mathrm{p}<0.05$.

\section{RESULTS}

Lower performances in divided attention were associated with difficulties in high-traffic intersections $(p=0.008)$, trouble seeing the signs in time to respond to them $(p=0.011)$ and problems in judging the speed or distance of an approaching vehicle $(p=0.029)$. Concerning TTA, greater VE judging the vehicle approaching at $50 \mathrm{~km} / \mathrm{h}$ was associated with difficulties overtaking other cars $(\mathrm{p}=0.030)$ and difficulties in seeing the signs in time to react to them $(p=0.029)$; higher CE judging the vehicle approaching at $70 \mathrm{~km} / \mathrm{h}$ was associated with difficulties in the speed or distance perception of an oncoming vehicle $(\mathrm{p}=0.029)$.

To understand the particular contributions of TTA and UFOV in explaining the two self-reported difficulties related simultaneously with both measures, a logistic regression was applied including them as covariates (along with age, gender and visual acuity). In both cases, UFOV 
and TTA remained in the final equation, revealing that they had an independent contribution for the specific driving difficulty detected.

Regarding the associations between UFOV and TTA measures, a calculated correlation coefficient (see Table 1) only revealed a low association between selective attention and CE judging a car approaching at $50 \mathrm{~km} / \mathrm{h}(\mathrm{p}=0,046$, Spearman's Rho=-0,326). This association means that older divers with greater $\mathrm{CE}$ had also greater selective attention difficulties.

Comparing the error measures between the two velocity paradigms used in TTA, a significant difference was found in CE when the velocity of the vehicle approaching was $50 \mathrm{~km} / \mathrm{h}$ and 70 $\mathrm{km} / \mathrm{h}$, respectively $(\mathrm{p}<0,001)$. These results showed that TTA estimated accuracy increased when the vehicle was traveling at higher speeds (see Figure 1).

Table 1. Spearman's rank correlation coefficient between TTA and UFOV ${ }^{\circledR}$ and between visual acuity and $\mathrm{UFOV}^{\circledR}$

\begin{tabular}{cccc}
\hline & Subtest1 & Subtest2 & Subtest3 \\
\hline AE50km/h & 0.008 & 0.142 & 0.091 \\
AE70km/h & -0.024 & 0.085 & -0.133 \\
CE50km/h & -0.010 & -0.115 & $-0.326^{*}$ \\
$\mathbf{C E 7 0 k m / h}$ & -0.073 & -0.092 & -0.258 \\
VE50km/h & -0.110 & 0.106 & -0.151 \\
VE70km/h & -0.041 & 0.110 & -0.288 \\
Visual Acuity & -0.282 & -0.307 & $-0.604^{*}$ \\
\hline
\end{tabular}

$* \mathrm{p}<0.05$

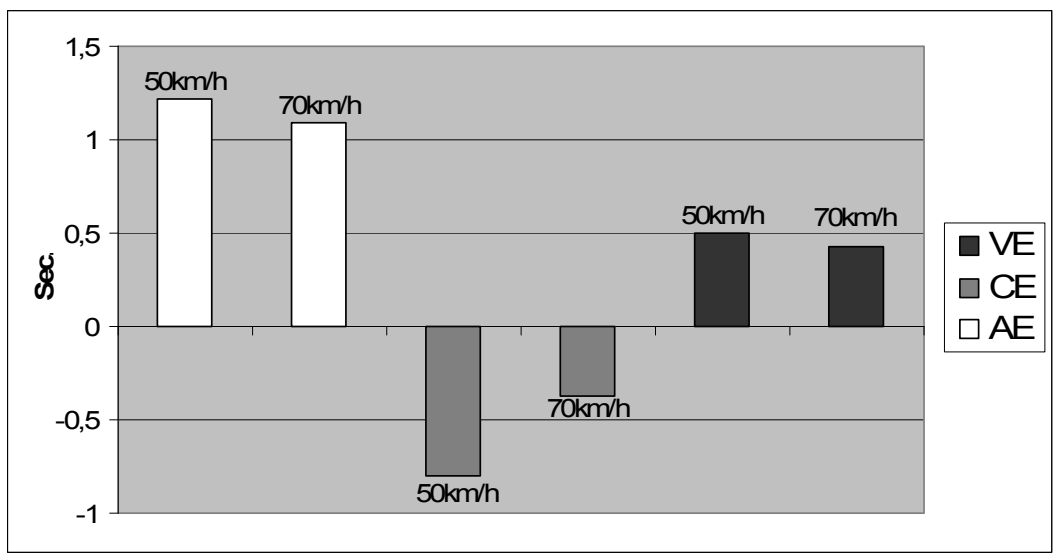

Figure 1. Error values for TTA measures at $50 \mathrm{~km} / \mathrm{h}$ and $70 \mathrm{~km} / \mathrm{h}$

\section{DISCUSSION}

Four self-reported difficulties were significantly associated with UFOV or TTA. Concerning UFOV, only subtest 2 of $\mathrm{UFOV}^{\circledR}$ (divided attention) was linked to driving difficulties:

difficulties in high-traffic intersections, trouble seeing the signs in time to respond to them and problems in judging the speed or distance of an approaching vehicle. All three difficulties were 
associated with poorer scores in subtest 2 of $\mathrm{UFOV}^{\circledR}$. The larger sensibility of this subtest in detecting problems related to driving was previously mentioned (Owsley et al., 1998), bringing out the idea that in settings where limited time is available, this should the prioritary UFOV ${ }^{\circledR}$ subtest selected. TTA measures were also significantly associated with three self-reported difficulties: trouble seeing the signs in time to respond, problems judging the speed or distance of an approaching vehicle and difficulties in overtaking other cars. In these cases, there was an association with greater CE or VE.

It seems that older drivers reveal more difficulties calculating other vehicles TTA. Staplin et al. (1993) in a series of complementary simulation and field studies, suggested that the perceptual basis of time-to-collision (concept equivalent to TTA) and traffic gap acceptance judgments, changes significantly with advancing adult age. Staplin and Lyles (1992) argue that age-related motion perception deficits combine to eliminate important cues needed for driving and may directly impact the safety of older drivers. Older females, in particular, appear to exhibit pronounced errors in estimating the TTA of approaching vehicles (Schiff et al., 1992).

There was a clear underestimation of TTA in our study, establishing a synchrony with the majority of other comparable works (Caird \& Hancock, 1994; Hesketh \& Godley, 2002; Horswill et al., 2005). That is, approaching vehicles were estimated to arrive sooner than they actually do. Participants were also able to estimate TTA with greater accuracy and less variability when the vehicle approached at higher velocity $(70 \mathrm{~km} / \mathrm{h}$ vs. $50 \mathrm{~km} / \mathrm{h})$ repeating former conclusions of other investigations (Horswill et al., 2005; Manser \& Hancock, 1996). Less accuracy or more variability in TTA, combined with other factors like longer times required to cross the road or perform a turning manoeuvre (Keskinen et al., 1998; Skaar et al., 2003), could explain why older drivers tend to be more conservative than younger adults when deciding to enter traffic, accepting larger gaps, and by this means trying to reduce the probability of a traffic accident (Skaar et al., 2003). In this context, relative underestimation of TTA might reflect some loss of perception capability, but on the another hand, could prove beneficial in terms of preventive behaviour, for example encouraging drivers to choose large gaps between successive oncoming vehicles (Hesketh \& Godley, 2002).

One of the objectives of this study was to examine the associations between UFOV and TTA measures. The results only revealed a low association between selective attention and CE judging a car approaching at $50 \mathrm{~km} / \mathrm{h}(\mathrm{p}=0,046$, Spearman's Rho=-0,326). This modest association is somewhat in agreement with Raghuram and Lakshminarayanan (2006), which found that the correlations between UFOV and Motion Index Scores (resulting from various perceptual tests including TTA) weren't significant.

In our study, limited to a relatively small sample size, we found evidence that TTA could be a relevant independent factor to detect some driving difficulties in older people. In the past, limitations have been pointed to with TTA concerning the eventual lack of visual realism and generalizability (Manser \& Hancock, 1996). In the current study, we use filmed footage of actual road situations rather then computer-generated scenes to increase the realism of the stimuli. This decision derives from the fact that the closer the visual task used in the laboratory is to a driving environment, the more successful the test would be to identify at-risk drivers (Raghuram \& Lakshminarayanan, 2006). In respect to speed perception, Recarte and Nunes (2005) studied the 
equivalence of TTA estimations performed in vehicle and using video images, indicating high equivalence results.

TTA probably involves primarily the local transformation of optical information through changes in the size of the image on the retina (Caird \& Hancock, 1994; Hesketh \& Godley, 2002; Manser \& Hancock, 1996). More recently, Horswill et al. (2005) suggested that other variables could contribute to explain the estimation of TTA, namely the dimension of the objects approaching (size-arrival effect). UFOV ${ }^{\circledR}$ test performance relies on higher-order cognitive abilities, as well as visual sensory function (Owsley et al., 1995). It's possible that individuals with intact visual sensory fields can also exhibit poor performance in UFOV (Owsley et al., 1995); in our study, only subtest 3 of $U_{F O V}^{\circledR}$ revealed a significant association with visual acuity.

\section{CONCLUSION}

Both TTA and UFOV seem to be important measures for detecting driving difficulties in older adults. However, correlations between UFOV and TTA were low, suggesting that they probably measure different aspects of visual information processing. The TTA and other measures of speed perception could be relevant for measuring specific aspects of visual information processing, in addition to other instruments like the UFOV ${ }^{\circledR}$ test. Further studies with older drivers should bring out this idea.

\section{REFERENCES}

Anstey, K., Wood, J., Lord, S., \& Walker, J.G. (2005). Cognitive, sensory and physical factors enanbling riving safety in older adults. Clinical Psychology Review, 25, 45-65.

Ball, K., \& Owsley, C. (1993). The useful field of view test: A new technique for evaluating age related declines in visual function. Journal of the American Optometric Association, 64(1), 71-79.

Caird, J.K., \& Hancock, P.A. (1994). The perception of arrival time for different oncoming vehicles at an intersection. Ecological Psychology, 6, 83-109.

Clay, O.J., Wadley, V.G., Edwards, J.D., Roth, D.L., Roenker, D.L., \& Ball., K.K. (2005). Cumulative meta-analysis of the relationship between useful field of view and driving performance in older adults: Current and future implications. Optometry and Visual Science, $82,724-731$.

Guerrier, J.H., Manivannan, P., \& Nair, S. (1999). The role of working memory, field dependence, visual search, and reaction time in the left turn performance of older female drivers. Applied Ergonomics, 30, 109-119.

Hesketh, B., \& Godley, S.T. (2002). A comparison of time estimations in driving with targetonly in motion, self-only in motion, and self-and-target in motion. Ecological Psychology, 14 (3), 111-125.

Horswill, M.S., Helman, S., Ardiles, P., \& Wann, J.P. (2005). Motorcycle accident risk could be inflated by a time to arrival illusion. Optometry and Vision Science, 82(8), 740-746. 
Keskinen, E., Ota, H., \& Katila, A. (1998). Older drivers fail in intersections: Speed discrepancies between older and younger mail drivers. Accident Analysis and Prevention, 30(3), 151-159.

Manser, M.P., \& Hancock, P.A. (1996). Influence of approach angle on estimates of Time-toContact. Ecological Psychology, 8(1), 71-99.

Mayhew, D.R., Simpson, H.M., \& Ferguson, S.A. (2006). Collisions involving senior drivers: high-risk conditions and locations. Traffic Injury Prevention, 7, 117-124.

McGwin, G., \& Brown, D.B. (1999). Characteristics of traffic crashes among young, middleaged, and older drivers. Accident Analysis and Prevention, 31 (3), 181-198.

McGwin, G., Sims. R.V., Pulley, L., \& Roseman, J.M. (2000). Relations among chronic medical conditions, medications, and automobile crashes in the elderly: a population-based casecontrol study. American Journal of Epidemiology, 152, 5, 424-431.

Owsley, C., Ball, K., \& Keeton, D.M. (1995). Relationship between visual sensitivity and target localization in older adults. Vision Research, 35, 579-587.

Owsley, C., Ball, K., McGwin, G. J., Sloane, M. E., Roenker, D. L., White, M. F., \& Overley,T. (1998). Visual processing impairment and risk of motor vehicle crash among older. JAMA: The Journal of the American Medical Association, 279(14): 1083-1088.

Owsley, C., Stalvey, B., Wells, J., \& Sloane, M. E. (1999). Older drivers and cataract: Driving habits and crash risk. Journals of Gerontology Series A: Biological Sciences and Medical Sciences, 54A (4), M203-M211.

Preusser, D.F., Williams, A.F., Ferguson, S.A., Ulmer, R.G., \& Weinstein, H.B. (1998). Fatal crash risk for older drivers at intersections. Accident Analysis and Prevention, 30(2), 151159.

Raghuram, A., \& Lakshminarayanan, V. (2006). Motion perceptions tasks as potential correlates to driving difficulty in the elderly. Journal of Modern Optics, 53(9), 1343-1362.

Recarte, M.A., Conchillo, A., \& Nunes, L.M. (2005). Estimation of arrival time in vehicle and video. Psicothema, 17(1), 112-117.

Schiff, W., Oldak, R., \& Shah, V. (1992). Aging persons' estimates of vehicular motion. Psychology and Aging, 7, 518-525.

Sims, R., McGwin, G., Allman, R., Ball, K., \& Owsley, C. (2000). Exploratory study of incident vehicle crashes among older drivers. Journals of Gerontology Series A: Biological Sciences and Medical Sciences, 55A(1), M22-M27.

Skaar, N., Rizzo, M., \& Stierman (2003). Traffic entry judgments by aging drivers. Proceedings of the Second International Driving Symposium on Human Factors in Driver Assessment, Training and Vehicle Design. Park City, Utah

Staplin, L., Lococo, K., \& Sim, J. (1993). Traffic Maneuver Problems of Older Drivers. Publication No. FHWA-RD-92-092, Federal Highway Administration, Washington, DC.

Staplin, L., \& Lyles, R.W. (1992). Age differences in motion perception and specific traffic maneuver problems. Transportation Research Record, 1325, 23-33. 